\title{
Logarithmic functional and reciprocity laws
}

\author{
Askold Khovanskii
}

\begin{abstract}
In this paper, we give a short survey of results related to the reciprocity laws over the field $\mathbb{C}$. We announce a visual topological proof of Parshin's multidimensional reciprocity laws over $\mathbb{C}$. We introduce the logarithmic functional, whose argument is an $n$-dimensional cycle in the group $\left(\mathbb{C}^{*}\right)^{n+1}$. It generalizes the usual logarithm, which can be considered as the zero-dimensional logarithmic functional. It also generalizes the one-dimensional logarithmic functional that is a natural extension of the functional introduced by Beilinson for a topological proof of the Weil reciprocity law over $\mathbb{C}$.
\end{abstract}

\section{One-dimensional case}

1.1. Weil reciprocity law. Let $\Gamma$ be a complete connected complex onedimensional manifold (in other words, $\Gamma$ is an irreducible complex algebraic curve). A local parameter $u$ near a point $a \in \Gamma$ is an arbitrary meromorphic function, whose order at $a$ is equal to one. The local parameter $u$ is a coordinate function in a small neighborhood of $a$. Let $\varphi$ be a meromorphic function on $\Gamma$ and let $\sum_{k \leq m} c_{m} u^{m}$ be its Laurent expansion at $a$. The leading monomial $\chi$ of $\varphi$ is the first nonzero term in the expansion, i.e. $\chi(u)=c_{k} u^{k}$. The leading monomial is defined for any meromorphic function $\varphi$ not identically equal to zero. For each pair of meromorphic functions $f, g$ on a curve $\Gamma$ not identically equal to zero and each point $a \in \Gamma$, one defines the Weil symbol $[f, g]_{a}$. It is a nonzero complex number given by the formula

$$
[f, g]_{a}=(-1)^{n m} a_{m}^{n} b_{n}^{-m},
$$

where $a_{m} u^{m}$ and $b_{n} u^{n}$ are the leading monomials of the functions $f$ and $g$ at $a$, with respect to the parameter $u$. The Weil symbol is defined with the help of the parameter $u$ but it does not depend on the choice of $u$. By definition, the Weil symbol depends multiplicatively on functions $f$ and $g$. The multiplicativity with respect to $f$ means that if $f=f_{1} f_{2}$, then $[f, g]_{a}=\left[f_{1}, g\right]_{a}\left[f_{2}, g\right]_{a}$. The multiplicativity with respect to $g$ is defined similarly. The Weil symbol of functions $f, g$ can differ from 1 only at points in the supports of the divisors of $f$ and $g$.

1991 Mathematics Subject Classification. Primary 11S31, Secondary 14M25.

Key words and phrases. Weil reciprocity law, multidimensional reciprocity laws, symbols, logarithmic functional.

Partially supported by Canadian Grant No. OGP0156833. 
The WeIL RECIPROCITY LAW. The product of the Weil symbols $[f, g]_{a}$ over all points a of the curve $\Gamma$ is equal to one

$$
\prod_{a \in X}[f, g]_{a}=1
$$

EXAMPLE. Take the Riemann sphere for $\Gamma$, an affine coordinate function $x$ for $f$, and a polynomial $P=a_{n} x^{n}+\cdots+a_{k} x^{k}$ of degree $n$ for $g$. By the reciprocity law,

$$
\prod x(a)=[x, P]_{0}^{-1}[x, P]_{\infty}^{-1}=(-1)^{-k} a_{k}^{-1}(-1)^{n} a_{n}=(-1)^{n-k} a_{n} / a_{k},
$$

where the product is over all nonzero roots $a$ of $P$. This formula coincides with the Vieta formula.

1.2. Toric Surfaces and the reciprocity law (see [1]). Consider a compact (possibly singular) toric surface $M$. Let $D$ be a zero-dimensional positive divisor in the union of one-dimensional orbits. Is there an algebraic curve on the surface $M$ that does not pass through zero-dimensional orbits and intersects onedimensional orbits at the given divisor $D$ ?

Let us fix an orientation in the plane of one-parameter subgroups of $\left(\mathbb{C}^{*}\right)^{2}$. Thus we fix a parameterization $\pi_{j}: \mathbb{C}^{*} \rightarrow M_{j}$ of each one-dimensional orbit $M_{j}$ in $M$. Consider the map $\pi: \bigcup_{j} M_{j} \rightarrow \mathbb{C}^{*}$, whose restriction to $M_{j}$ equals to $\pi_{j}^{-1}$.

THEOREM. If $D=\sum k_{i} a_{i}$ is the divisor of the intersection of a curve not passing through zero-dimensional orbits of $M$ with the union of one-dimensional orbits of $M$, then

$$
\prod(-\pi(a))^{k_{i}}=1
$$

Sketch OF THE PROOF. Each curve in $\left(\mathbb{C}^{*}\right)^{2}$ is given by an equation $P=0$, where $P$ is a Laurent polynomial. Let $\Delta$ be the Newton polygon of $P$. On each side $\mathbf{n}_{j}$ of $\Delta$, a polynomial $P_{\mathbf{n}_{j}}$ in one variable is written. By the Vieta formula, the product of all nonzero roots of all polynomials $P_{\mathbf{n}_{j}}$ is equal to 1 .

The reciprocity law follows from the previous theorem (see [1]).

1.3. Topological proof of the reciprocity law (see [2 - 4]). Consider a complex algebraic curve $\Gamma$ and a pair $f, g$ of nonzero meromorphic functions on $\Gamma$. Let $A$ be the union of the supports of the principle divisors $(f),(g)$, and let $U$ be $\Gamma \backslash A$. With the pair $f, g$, Beilinson associated a certain cohomology class $[f, g] \in H^{1}\left(U, \mathbb{C}^{*}\right)$. One can define this class using the Beilinson Integral.

Definition. The Beilinson Integral against a loop $\gamma: I \rightarrow U, \gamma(0)=\gamma(1)$, for a pair of analytic functions $f: U \rightarrow \mathbb{C}^{*}, g: U \rightarrow \mathbb{C}^{*}$ is an element $I_{\gamma}(f, g)$ of the group $\mathbb{C} / \mathbb{Z}$ defined by the formula

$$
I_{\gamma}(f, g)=\left(\frac{1}{2 \pi i}\right)^{2} \int_{I} \ln \left(\gamma^{*} f\right) \frac{d\left(\gamma^{*} g\right)}{\left(\gamma^{*} g\right)}-\frac{1}{2 \pi i} \operatorname{deg}_{\gamma}(f) \ln g(\gamma(1)),
$$

where $\ln \left(\gamma^{*} f\right)$ is a continuous branch of the multi-valued function $\ln \left(\gamma^{*} f\right)$ over the interval $0<t<1$, and $\operatorname{deg}_{\gamma}(f)$ is the mapping degree of the map $\frac{f}{|f|}: \gamma(I) \rightarrow S^{1}$. 
One can prove that the Beilinson integral has the following properties. It is invariant under orientation preserving reparameterizations of the loop $\gamma$. It is skew symmetric, i.e. $I_{\gamma}(f, g)=-I_{\gamma}(g, f)$, and additive, i.e. $I_{\gamma}\left(f_{1} f_{2}, g\right)=I_{\gamma}\left(f_{1}, g\right)+$ $I_{\gamma}\left(f_{2}, g\right) ; I_{\gamma}\left(f, g_{1} g_{2}\right)=I_{\gamma}\left(f, g_{1}\right)+I_{\gamma}\left(f, g_{1}\right)$. On the diagonal, it is related to the mapping degree: $I_{\gamma}(f, f)=\frac{1}{2} \operatorname{deg}_{\gamma}(f)$.

One can slightly extend the previous definition and define the Beilinson integral $I_{\gamma}(f, g)$ against a linear combination $\gamma=\sum k_{i} \gamma_{i}$ of loops $\gamma_{i}$ with integer coefficients $k_{i}$.

TheOREM. The Beilinson integral $I_{\gamma}(f, g)$ depends only on the homology class of the cycle $\gamma=\sum k_{i} \gamma_{i}$ in $U$ and defines an element in $H^{1}(U, \mathbb{C} / \mathbb{Z})$.

TheOREM. The number $[f, g, \gamma]=\exp \left(2 \pi i I_{\gamma}(f, g)\right)$ depends only on the homology class of the cycle $\gamma=\sum k_{i} \gamma_{i}$ in $U$ and defines an element in $H^{1}\left(U, \mathbb{C}^{*}\right)$. It is skew-symmetric $[f, g, \gamma]=[g, f, \gamma]^{-1}$ and multiplicative $\left[f_{1} f_{2}, g, \gamma\right]=\left[f_{1}, g, \gamma\right]$. $\left[f_{2}, g, \gamma\right] ;\left[f, g_{1} g_{2}, \gamma\right]=\left[f, g_{1}, \gamma\right]\left[f, g_{2}, \gamma\right]$.

THEOREM. Consider a small ball $B_{a}$ centered at a point $a \in X$. Let $\gamma$ be its boundary $\gamma=\partial B$. Then $[f, g, \gamma]$ is equal to the Weil symbol $[f, g]_{a}$.

Now let us give a topological proof of the reciprocity law. Let $A$ be the union of supports of principle divisors $(f),(g)$, and let $U$ be $\Gamma \backslash A$. Let $B$ be the union of small balls $B_{a}$ centered at all points $a \in A$ and let $\gamma=\sum_{a \in A} \partial B_{a}$ be the boundary of the domain $B$. Then $\gamma=0$ in $H_{1}(U, \mathbb{Z})$ and $[f, g, \gamma]=\prod_{a \in A}[f, g]_{a}=1$. The reciprocity law is proved.

\section{Product of roots of a system of equations with generic Newton polyhedra (see [5])}

Consider a system of equations

$$
P_{1}=\cdots=P_{n}=0
$$

in $\left(\mathbb{C}^{*}\right)^{n}$, where $P_{1}, \ldots, P_{n}$ are Laurent polynomials. Let $\Delta_{1}, \ldots, \Delta_{n}$ be the Newton polyhedra of $P_{1}, \ldots, P_{n}$.

Problem: Compute the product in the group $\left(\mathbb{C}^{*}\right)^{n}$ of roots of system (1) assuming that the collection of Newton polyhedra $\Delta_{1}, \ldots, \Delta_{n}$ is generic.

2.1. Developed sets of polyhedra. Let $\Delta_{1}, \ldots, \Delta_{n}$ be convex polyhedra in $\mathbb{R}^{n}$, and let $\Delta$ be their Minkowski sum. Each face $\Gamma$ of the polyhedron $\Delta$ can be uniquely represented as a sum

$$
\Gamma=\Gamma_{1}+\cdots+\Gamma_{n}
$$

where $\Gamma_{i}$ is a face of $\Delta_{i}$.

A collection of $n$ polyhedra $\Delta_{1}, \ldots, \Delta_{n}$ is called developed if for each face $\Gamma$ of the polyhedron $\Delta$, at least one of the terms $\Gamma_{i}$ in its decomposition is a vertex.

For a developed collection of polyhedra $\Delta_{1}, \ldots, \Delta_{n}$, a map $f: \partial \Delta \rightarrow \partial \mathbb{R}_{+}^{n}$ of the boundary of $\Delta=\sum \Delta_{i}$ into the boundary of the positive octant is called characteristic if the component $f_{i}$ of the map $f=\left(f_{1}, \ldots, f_{n}\right)$ vanishes precisely on the faces $\Gamma$, for which the $i$-th term $\Gamma_{i}$ in the decomposition is a point (a vertex of the polyhedron $\Delta_{i}$ ). The preimage of the origin under the characteristic map is precisely the set of all vertices of the polyhedron $\Delta$. 
The combinatorial coefficient $C_{A}$ of a vertex $A$ of $\Delta$ is the local degree of the germ

$$
f:(\partial \Delta, A) \rightarrow\left(\partial \mathbb{R}_{+}^{n}, 0\right)
$$

of the characteristic map restricted to the boundary $\partial \Delta$ of $\Delta$.

2.2. Parshin symbols see [5 - 9]. Consider $n+1$ monomials $c_{1} \mathbf{x}^{\mathbf{k}_{1}}, \ldots$, $c_{n+1} \mathbf{x}^{\mathbf{k}_{n+1}}$ in $n$ complex variables, where $c_{i} \in \mathbb{C}^{*}, \mathbf{x}=\left(x_{1}, \ldots, x_{n}\right), \mathbf{k}_{i} \in(\mathbb{Z})^{n}, \mathbf{k}_{i}=$ $\left(k_{i, 1}, \ldots, k_{i, n}\right), c_{i} \mathbf{x}^{\mathbf{k}_{i}}=c_{i} x_{1}^{k_{i, 1}} \cdot \ldots \cdot x_{n}^{k_{i, n}}$. The Parshin Symbol $\left[c_{1} \mathbf{x}^{\mathbf{k}_{1}}, \ldots, c_{n+1} \mathbf{x}^{\mathbf{k}_{n+1}}\right]$ of the sequence $c_{1} \mathbf{x}^{\mathbf{k}_{1}}, \ldots, c_{n+1} \mathbf{x}^{\mathbf{k}_{n+1}}$ is equal by definition to

$$
\begin{gathered}
(-1)^{D\left(\mathbf{k}_{1}, \ldots, \mathbf{k}_{n+1}\right)} c_{1}^{-\operatorname{det}\left(\mathbf{k}_{2}, \ldots, \mathbf{k}_{n+1}\right)} \ldots c_{n+1}^{(-1)^{n+1} \operatorname{det}\left(\mathbf{k}_{1}, \ldots, \mathbf{k}_{n}\right)}= \\
=(-1)^{D\left(\mathbf{k}_{1}, \ldots, \mathbf{k}_{n+1}\right)} \exp \left(-\operatorname{det}\left(\begin{array}{cccc}
\ln c_{1} & k_{1,1} & \ldots & k_{1, n+1} \\
\vdots & \vdots & & \vdots \\
\ln c_{n+1} & k_{n+1,1} & \ldots & k_{n+1, n+1}
\end{array}\right)\right),
\end{gathered}
$$

where $D:\left(\mathbb{Z}^{n}\right)^{n+1} \rightarrow \mathbb{Z} / 2 \mathbb{Z}$ is a function (see [9]) with the following properties. The function $D$ depends only on the images $\pi\left(\mathbf{k}_{1}\right), \ldots, \pi\left(\mathbf{k}_{n+1}\right) \in(\mathbb{Z} / 2 \mathbb{Z})^{n}$ of the exponents $\mathbf{k}_{1}, \ldots, \mathbf{k}_{k+1}$ under the natural projection $\pi:(\mathbb{Z})^{n} \rightarrow(\mathbb{Z} / 2 \mathbb{Z})^{n}$. It is the only nonzero multilinear function of $n+1$ vectors in the $n$-dimensional space over the field $\mathbb{Z} / 2 \mathbb{Z}$ that is invariant under all linear transformations and vanishes whenever the rank of $n+1$ vectors is less than $n$.

ExAmple. The Parshin symbol $\left[c_{1} x^{k_{1}}, c_{2} x^{k_{2}}\right]$ of two monomials $c_{1} x^{k_{1}}, c_{2} x^{k_{2}}$ in one variable $x$ equals to $(-1)^{k_{1} k_{2}} c_{1}^{-k_{2}} c_{1}^{k_{1}}$. Thus it is equal to the Weil symbol $\left[c_{1} x^{k_{1}}, c_{2} x^{k_{2}}\right]_{0}$ of these monomials at the origin $x=0$.

By definition, the Parshin symbol is skew-symmetric, for example,

$$
\left[c_{1} \mathbf{x}^{\mathbf{k}_{1}}, c_{2} \mathbf{x}^{\mathbf{k}_{2}}, \ldots, c_{n+1} \mathbf{x}^{\mathbf{k}_{n+1}}\right]=\left[c_{2} \mathbf{x}^{\mathbf{k}_{2}}, c_{1} \mathbf{x}^{\mathbf{k}_{1}}, \ldots, c_{n+1} \mathbf{x}^{\mathbf{k}_{n+1}}\right]^{-1},
$$

and multiplicative, for example, if $c_{1} \mathbf{x}^{\mathbf{k}_{1}}=a_{1} b_{1} \mathbf{x}^{\mathbf{l}_{1}+\mathbf{m}_{1}}$, then

$$
\left[c_{1} \mathbf{x}^{\mathbf{k}_{1}}, \ldots, c_{n+1} \mathbf{x}^{\mathbf{k}_{n+1}}\right]=\left[a_{1} \mathbf{x}^{\mathbf{l}_{1}}, \ldots, c_{n+1} \mathbf{x}^{\mathbf{k}_{n+1}}\right]\left[b_{1} \mathbf{x}^{\mathbf{m}_{1}}, \ldots, c_{n+1} \mathbf{x}^{\mathbf{k}_{n+1}}\right] .
$$

2.3. The value of a character at the product of roots. Let $\chi_{\mathbf{k}}:\left(\mathbb{C}^{*}\right)^{n} \rightarrow$ $\mathbb{C}^{*}$ be the character corresponding to a point $\mathbf{k} \in \mathbb{Z}^{n}$, i.e. for $\mathbf{k}=\left(k_{1}, \ldots, k_{n}\right)$ and $\mathbf{x}=\left(x_{1}, \ldots, x_{n}\right)$ the character $\chi_{\mathbf{k}}(\mathbf{x})$ is equal to $x_{1}^{k_{1}} \ldots x_{n}^{k_{n}}$.

With every vertex $A$ of the polyhedron $\Delta=\Delta_{1}+\cdots+\Delta_{n}$, where $\Delta_{i}$ is the Newton polyhedron of a Laurent polynomial $P_{i}$, we associate a number $\left[P_{1}, \ldots, P_{n}, \chi_{\mathbf{k}}\right]_{A} \in \mathbb{C}^{*}$ in the following way: let $A=A_{1}+\cdots+A_{n}$ be the decomposition of the vertex $A \in \Delta, A_{i} \in \Delta_{i}$. Assume that the vertex $A_{i}$ of the polyhedron $\Delta_{i}$ corresponds to a monomial $c_{i} \mathbf{x}^{\mathbf{k}_{i}}$ of the polynomial $P_{i}$. Then the number $\left[P_{1}, \ldots, P_{n}, \chi_{\mathbf{k}}\right]_{A}$ is by definition the Parshin symbol $\left[c_{1} \mathbf{x}^{\mathbf{k}_{1}}, \ldots, c_{n} \mathbf{x}^{\mathbf{k}_{n}}, \chi_{\mathbf{k}}\right]$.

THEOREM. For a system of equations (1), the value of the character $\chi_{\mathbf{k}}$ at the product $M\left(P_{1}, \ldots, P_{n}\right)$ of roots is given by

$$
\chi_{\mathbf{k}}\left(M\left(P_{1}, \ldots, P_{n}\right)\right)=\prod_{A \in \Delta}\left(\left[P_{1}, \ldots, P_{n}, \chi_{\mathbf{k}}\right]_{A}\right)^{(-1)^{n} C_{A}},
$$

where the product is over all vertices $A$ of the polyhedron $\Delta=\Delta_{1}+\cdots+\Delta_{n}$, and $C_{A}$ is the combinatorial coefficient at $A$. 
As an application of the theorem, one can compute all coordinates in $\left(\mathbb{C}^{*}\right)^{n}$ of the product $M\left(P_{1}, \ldots, P_{n}\right)$ of roots: each coordinate $x_{i}$ can be considered as the character $\chi_{\mathbf{k}}$ for $\mathbf{k}=e_{i}$, where the vector $e_{i}$ is the $i$-th vector in the standard basis of the lattice $(\mathbb{Z})^{n}$. The proof of the theorem (see [5]) is based on simple geometry and does not use Parshin theory.

\section{Multidimensional case}

Parshin generalized the Weil reciprocity law to a multidimensional case (see $[6-9])$. Here we discuss this result and its topological proof due to BrylinskiMcLaughlin (see [10]).

3.1. Generalized Points, Parameters, Symbols, Flags and Reciprocity Laws (see [6 - 8]). Let $X$ be a complete irreducible complex $n$-dimensional algebraic variety (possibly very singular). A sequence $Y_{0} \stackrel{\pi_{0}}{\longrightarrow} Y_{1} \stackrel{\pi_{1}}{\longrightarrow} \ldots \stackrel{\pi_{n-1}}{\longrightarrow} Y_{n} \stackrel{\pi_{n}}{\longrightarrow}$ $X$ consisting of complete normal irreducible $i$-dimensional algebraic varieties $Y_{i}$ with $i=0, \ldots, n$, equipped with a collection of maps $\pi_{0}, \ldots \pi_{n}$ is called a generalized point of the variety $X$ if for $i=0, \ldots, n-1$, the map $\pi_{i}: Y_{i} \rightarrow Y_{i+1}$ is a normalization of the image $\pi_{i}\left(Y_{i}\right) \subset Y_{i+1}$ and $\pi_{n}: Y_{n} \rightarrow X$ is a normalization of $X$. We identify two generalized points $G_{1}=\left(Y_{0} \stackrel{\pi_{0}}{\longrightarrow} Y_{1} \stackrel{\pi_{1}}{\longrightarrow} \ldots \stackrel{\pi_{n-1}}{\longrightarrow} Y_{n} \stackrel{\pi_{n}}{\longrightarrow} X\right)$ and $G_{2}=\left(Z_{0} \stackrel{\rho_{0}}{\longrightarrow} Z_{1} \stackrel{\rho_{1}}{\longrightarrow} \ldots \stackrel{\rho_{n-1}}{\longrightarrow} Z_{n} \stackrel{\rho_{n}}{\longrightarrow} X\right)$ if for $i=0, \ldots, n$, there are isomorphisms $\tau_{i}: Z_{i} \rightarrow Y_{i}$ such that $\pi_{i} \circ \tau_{i}=\tau_{i+1} \circ \rho_{i}$.

Let us give an inductive definition for a set of parameters near a generalized point. A collection of rational functions $u_{1}, \ldots, u_{n}$ on $X$ is called a set of parameters near a generalized point $G=\left(Y_{0} \stackrel{\pi_{0}}{\longrightarrow} Y_{1} \stackrel{\pi_{1}}{\longrightarrow} \ldots \stackrel{\pi_{n-1}}{\longrightarrow} Y_{n} \stackrel{\pi_{n}}{\longrightarrow} X\right)$ if the following conditions hold:

1) each of the rational functions $\pi_{n}^{*} u_{1}, \ldots, \pi_{n}^{*} u_{n}$ on $Y_{n}$ has no poles on $X_{n-1}=$ $\pi_{n-1}\left(Y_{n-1}\right)$;

2) the principal divisor $\left(\pi_{n}^{*} u_{n}\right)$ in the normal variety $Y_{n}$ contains the subvariety $X_{n-1}$ with coefficient 1 ;

$3)$ if $n>1$, then the set of restrictions of $\pi_{n}^{*} u_{1}, \ldots, \pi_{n}^{*} u_{n-1}$ to $X_{n-1}$ is a set of parameters for the generalized point $\tilde{G}=\left(Y_{0} \stackrel{\pi_{0}}{\longrightarrow} Y_{1} \stackrel{\pi_{1}}{\longrightarrow} \ldots \stackrel{\pi_{n-2}}{\longrightarrow} Y_{n-1} \stackrel{\pi_{n-1}}{\longrightarrow}\right.$ $\left.X_{n-1}\right)$ on the $(n-1)$-dimensional variety $X_{n-1}$.

With a rational function $f$ and a generalized point $G$ one can associate the leading monomial $f_{G}$ using a set of parameters near $G$. Let us give an inductive definition of the leading monomial. Let the order of the function $\pi_{n}^{*} f$ at $X_{n-1}$ be $k_{n}$. Then the restriction $\varphi$ of $\left(\pi_{n}^{*} f\right) u^{-k_{n}}$ to $X_{n-1}$ is well-defined. Consider the generalized point $\tilde{G}=\left(Y_{0} \stackrel{\pi_{0}}{\longrightarrow} Y_{1} \stackrel{\pi_{1}}{\longrightarrow} \ldots \stackrel{\pi_{n-2}}{\longrightarrow} Y_{n-1} \stackrel{\pi_{n-1}}{\longrightarrow} X_{n-1}\right)$ of the $(n-1)$ dimensional variety $X_{n-1}$. Let $c\left(\pi_{n}^{*} u_{1}\right)^{k_{1}} \ldots\left(\pi_{n}^{*} u_{n-1}\right)^{k_{n-1}}$ be the leading monomial of $\varphi$ at the generalized point $\tilde{G}$ with parameters $\left(\pi_{n}^{*} u_{1}\right), \ldots,\left(\pi_{n}^{*} u_{n-1}\right)$. Then, by definition, the leading monomial $f_{G}$ is equal to $c u_{1}^{k_{1}} \ldots u_{n-1}^{k_{n-1}} u_{n}^{k_{n}}$.

Let $f_{1}, \ldots, f_{n+1}$ be a sequence of $(n+1)$ rational functions on an $n$-dimensional algebraic variety $X$. With a generalized point $G$, one can associate the Parshin symbol $\left[f_{1}, \ldots, f_{n+1}\right]_{G}$ : by definition, it is equal to the Parshin symbol of a sequence of leading monomials of the functions with respect to a set of parameters near the generalized point $G$. One can prove that the Parshin symbol is independent of a set of parameters and depends only on the sequence of rational functions $f_{1}, \ldots, f_{n+1}$ and on the generalized point $G$. 
Consider a flag $F=\left(C_{0} \subset C_{1} \subset \cdots \subset C_{n-1}\right)$ in $X$ consisting of complete irreducible $k$-dimensional algebraic subvarieties $C_{k}$ of $X$ with $k=0,1, \ldots, n-1$. A generalized point $G=\left(Y_{0} \stackrel{\pi_{0}}{\longrightarrow} Y_{1} \stackrel{\pi_{1}}{\longrightarrow} \ldots \stackrel{\pi_{n-1}}{\longrightarrow} Y_{n} \stackrel{\pi_{n}}{\longrightarrow} X\right)$ is a generalized point over the flag $F$ if for $j=0, \ldots, n-1$, the image $\tilde{\pi}_{j}\left(Y_{j}\right)$ of $Y_{j}$ under the map $\tilde{\pi}_{j}=\pi_{n} \circ \pi_{n-1} \circ \cdots \circ \pi_{j}$ is equal to $C_{j}$. Over each flag $F$, there are finitely many different generalized points. Consider a flag $F=C_{0} \subset \cdots \subset C_{n-1}$ on $X$ and take a collection $f_{1}, \ldots, f_{n+1}$ of rational functions on $X$. The Parshin symbol $\left[f_{1}, \ldots, f_{n+1}\right]_{F}$ of the collection $f_{1}, \ldots, f_{n+1}$ at the flag $F$ is by definition the product of $\prod\left[f_{1}, \ldots, f_{n+1}\right]_{G}$ over all generalized points $G$ over the flag $F$.

ExAmPLE. Let $X$ be an irreducible algebraic curve. With a generalized point $G=\left(Y_{0} \stackrel{\pi_{0}}{\longrightarrow} Y_{1} \stackrel{\pi_{1}}{\longrightarrow} X\right)$ in $X$, one can associate the flag $F=(a)$ in $X$, where $a=\pi_{1} \circ \pi_{0}\left(Y_{0}\right)$, and the point $b=\pi_{0}\left(Y_{0}\right)$ on the normalization $Y_{1}$ of the curve $X$. A rational function $u$ on $X$ is a parameter near $G$ if $\pi_{1}^{*} u$ is a parameter near $b$ on $Y_{1}$. For a pair of rational functions $f_{1}, f_{2}$ on $X$, the Parshin symbol $\left[f_{1}, f_{2}\right]_{G}$ coincides with the Weil symbol $\left[\pi_{1}^{*} f_{1}, \pi_{1}^{*} f_{2}\right]_{b}$. The Parshin symbol $\left[f_{1}, f_{2}\right]_{F}$ at the flag $F$ is equal to the product $\prod\left[\pi_{1}^{*} f_{1}, \pi_{1}^{*} f_{2}\right]_{c}$ over all points $c \in \pi_{1}^{-1} a$.

Fix a flag $L=\left(C_{0} \subset \cdots \subset C_{n-1}\right)$ on $X$. For each $0<i<n$, denote by $\Psi^{i}(L)$ the set of all flags $F=\left(\tilde{C}_{0} \subset \cdots \subset \tilde{C}_{n-1}\right)$, where $\tilde{C}_{j}=C_{j}$ if $j \neq i$, and $\tilde{C}_{i}$ is any $i$-dimensional irreducible subvariety such that $C_{i-1} \subset \tilde{C}_{i} \subset C_{i+1}$. Denote by $\Psi^{0}(L)$ the set of all flags $F=\tilde{C}_{0} \subset C_{1} \cdots \subset C_{n-1}$ such that $\tilde{C}_{0}$ is a point in $C_{1}$.

PARSHIN'S RECIPROCITY LAWS (SEE $[6-8]$ ). Fix a collection $f_{1}, \ldots, f_{n+1}$ of rational functions on $X$, a flag $L$ in $X$ and a number $0 \leq i<n$. Then the symbol $\left[f_{1}, \ldots, f_{n+1}\right]_{F}$ is different from 1 for only finitely many flags $F \in \Psi^{i}(L)$, and the following relation holds

$$
\prod_{F \in \Psi^{i}(L)}\left[f_{1}, \ldots, f_{n+1}\right]_{F}=1 .
$$

3.2. The Brylinski-McLaughlin topological version of the Parshin theory (see $[\mathbf{1 0}])$. Consider a sequence $f_{1}, \ldots, f_{n+1}$ of $(n+1)$ rational functions on an $n$-dimensional complete complex algebraic variety $X$. Let $A$ be the union of the supports of the principle divisors $\left(f_{1}\right), \ldots,\left(f_{n+1}\right)$ and of the singular locus $S(X)$ of the variety $X$. Denote by $U$ the domain $X \backslash A$.

Brylinski and McLaughlin defined a certain cohomology class $\left(f_{1}, \ldots, f_{n+1}\right) \in$ $H^{n}\left(U, \mathbb{C}^{*}\right)$. The class $\left(f_{1}, \ldots, f_{n+1}\right)$ is skew symmetric in $f_{1}, \ldots, f_{n+1}$ and is multiplicative in each argument.

Let $F=\left(C_{0} \subset C_{1} \subset \cdots \subset C_{n-1}\right)$ be a flag of irreducible complete algebraic subvarieties of $X$ such that $\operatorname{dim} C_{k}=k$. With the flag $F$, Brylinski and McLaughlin associated a flag-localized homology class $\gamma_{F} \in H_{n}(U, \mathbb{Z})$. They had proved the following results.

Theorem (Topological Reciprocity LAWs). Fix a flag $L$ in $X$ and $a$ number $0 \leq i<n$. Then the flag-localized homology class $\gamma_{F} \in H_{n}(U, \mathbb{Z})$ is different from zero for only finitely many flags $F \in \Psi^{i}(L)$, and in the group $H_{n}(U, \mathbb{Z})$, the following relation holds

$$
\sum_{F \in \Psi^{i}(L)} \gamma_{F}=0 .
$$


TheOREM. The Parshin symbol $\left[f_{1}, \ldots, f_{n+1}\right]_{F}$ can be obtained by the pairing of the cohomology class $\left(f_{1}, \ldots, f_{n+1}\right) \in H^{n}\left(U, \mathbb{C}^{*}\right)$ with the flag-localized homology class $\gamma_{F} \in H_{n}(U, \mathbb{Z})$.

Using these results one can immediately obtain Parshin's reciprocity laws. So Brylinski and McLaughlin gave a topological proof of the multidimensional reciprocity laws over complex numbers and found a topological generalization of Parshin symbols. Their topological constructions make a heavy use of sheaf theory and are not visual at all.

The search for a formula for the product of the roots of a system of equations (see [5]) convinced me that, over complex number, there should be an intuitive geometric explanation of the Parshin symbols and reciprocity laws. The multidimensional logarithmic functional provides such an explanation.

\section{A Logarithmic Functional (see [11])}

4.1. Definitions and examples. Consider the group $\left(\mathbb{C}^{*}\right)^{n+1}$ with coordinate functions $x_{1}, \ldots, x_{n+1}$. The group $\left(\mathbb{C}^{*}\right)^{n+1}$ is homotopy equivalent to the torus $T^{n+1} \subset\left(\mathbb{C}^{*}\right)^{n+1}$ defined by equations $\left|x_{1}\right|=\cdots=\left|x_{n+1}\right|=1$. On the group $\left(\mathbb{C}^{*}\right)^{n+1}$, there is a remarkable $(n+1)$-form

$$
\omega=\left(\frac{1}{2 \pi i}\right)^{n+1} \frac{d x_{1}}{x_{1}} \wedge \cdots \wedge \frac{d x_{n+1}}{x_{n+1}} .
$$

Restriction of the form $\omega$ to the torus $T^{n+1}$ is the real $(n+1)$-form

$$
\left.\omega\right|_{T^{n+1}}=\frac{1}{(2 \pi)^{n}} d\left(\arg x_{1}\right) \wedge \cdots \wedge d\left(\arg x_{n+1}\right) .
$$

The integral of $\omega$ over the torus $T^{n+1}$ oriented by the form $\left.\omega\right|_{T^{n+1}}$ is equal to 1 .

Denote by $E$ the subset of $\left(\mathbb{C}^{*}\right)^{n+1}$ consisting of all points $\left(x_{1}, \ldots, x_{n+1}\right)$, one of whose coordinates is equal to 1 , i.e. $\left(x_{1}, \ldots, x_{n+1}\right) \in E \Leftrightarrow\{\exists j, 1 \leq j \leq n+1\}$ : $\left\{x_{j}=1\right\}$.

Let $X$ be an $n$-dimensional simplicial complex and let $\gamma=\sum k_{j} \Delta_{j}$, where $k_{j} \in \mathbb{Z}$ and $\Delta_{j}$ is an oriented $n$-dimensional cell in $X$, be an $n$-dimensional cycle, i.e. $\partial \gamma=0$. Consider a piecewise-smooth mapping $\mathbf{f}: X \rightarrow\left(\mathbb{C}^{*}\right)^{n+1}$, $\mathbf{f}=\left(f_{1}, \ldots, f_{n+1}\right)$, of the complex $X$ into the group $\left(\mathbb{C}^{*}\right)^{n+1}$.

The logarithmic functional is the functor that takes a map $\mathbf{f}: X \rightarrow\left(\mathbb{C}^{*}\right)^{n+1}$ and an $n$-dimensional cycle $\gamma=\sum k_{j} \Delta_{j}$ on $X$ to the element $\ln (\mathbf{f}, \gamma)$ of $\mathbb{C} / \mathbb{Z}$ defined by the formula

$$
\ln (\mathbf{f}, \gamma)=\int_{\sigma} \omega
$$

where $\sigma$ is an $(n+1)$-dimensional chain in $\left(\mathbb{C}^{*}\right)^{n+1}$, whose boundary $\partial \sigma$ is equal to the difference of the image $\mathbf{f}_{*}(\gamma)$ of $\gamma$ under the map $\mathbf{f}_{*}$ and some cycle $\gamma_{1}$ lying in $E$.

The logarithmic functional has the following obvious properties:

1) The value of the logarithmic functional is a well-defined element of the group $\mathbb{C} / \mathbb{Z}$.

2) The logarithmic functional depends skew-symmetrically on the components of the map $\mathbf{f}$, for example

$$
\ln \left(f_{1}, f_{2}, \ldots, f_{n+1}, \gamma\right)=-\ln \left(f_{2}, f_{1}, \ldots, f_{n+1}, \gamma\right)
$$


3) The logarithmic functional depends multiplicatively on the components of the map $\mathbf{f}$, for example,

$$
\ln \left(\varphi_{1} \psi_{1}, f_{2}, \ldots, f_{n+1}, \gamma\right)=\ln \left(\varphi_{1}, f_{2}, \ldots, f_{n+1}, \gamma\right)+\ln \left(\psi_{1}, f_{2}, \ldots, f_{n+1}, \gamma\right)
$$

EXAMPle (LOGARITHMiC FUNCTION AS LOGARITHMIC FUNCTIONAL). Let $X$ be a point $\{a\}$ and $\gamma$ the point $\{a\}$ with coefficient 1 . Then for a map $\mathbf{f}: X \rightarrow \mathbb{C}^{*}$, we have

where $f=\mathbf{f}$.

$$
\ln (\mathbf{f}, \gamma)=\frac{1}{2 \pi i} \ln f(a)
$$

ExAmple (The Beilinson integral as the LOGARITHMiC FUnCTIONAL $[3])$. Consider a complex algebraic curve $X$ and two nonzero meromorphic functions $f$ and $g$ on $X$. Let $A$ be the union of the divisors $(f)$ and $(g)$, and let $U$ be $X \backslash A$. Take a loop $\gamma$ in $U$. Then for a map $(f, g): \gamma \rightarrow\left(\mathbb{C}^{*}\right)^{2}$, we have

$$
\ln (\mathbf{f}, \gamma)=I_{\gamma}(f, g)
$$

where $\mathbf{f}=(f, g)$.

4.2. Topology and the logarithmic functional. The logarithmic functional has the following topological property. Let $M$ be a real manifold and f : $M \rightarrow\left(\mathbb{C}^{*}\right)^{n+1}$ a smooth map. An $n$-dimensional cycle $\tilde{\gamma}$ on $M$ can be considered as the image under a piecewise smooth map $\phi: X \rightarrow M$ of some $n$-dimensional cycle $\gamma$ on some $n$-dimensional complex $X$. With the cycle $\tilde{\gamma}$ on $M$, associate the element $\ln (\mathbf{f} \circ \phi, \gamma)$ of $\mathbb{C} / \mathbb{Z}$. The $n$-dimensional co-chain thus obtained gives an $n$ dimensional cohomology class of $M$ with coefficients in the group $\mathbb{C} / \mathbb{Z}$ if and only if $\mathbf{f}^{*} \omega \equiv 0$.

THEOREM. If the form $\mathbf{f}^{*} \omega$ is identically equal to zero on $M$, then the functional that takes a cycle $\phi: \gamma \rightarrow M$ to the element $\ln (\mathbf{f} \circ \phi, \gamma) \in \mathbb{C} / \mathbb{Z}$ is a cohomology class in $H^{n}(M, \mathbb{C} / \mathbb{Z})$.

MAin ExAmple. Let $X$ be an $n$-dimensional complex algebraic variety, $f_{1}, \ldots$, $f_{n+1}$ a sequence of $(n+1)$ rational functions on $X$, and $S(X)$ the singular locus of $X$. Consider the manifold $M=X \backslash\left(\left(f_{1}\right) \cup \cdots \cup\left(f_{n+1}\right) \cup S(X)\right)$ and the map $\mathbf{f}: M \rightarrow\left(\mathbb{C}^{*}\right)^{n+1}, \mathbf{f}=\left(f_{1}, \ldots, f_{n+1}\right)$. Then $\mathbf{f}^{*} \omega \equiv 0$. So, by the theorem, $\mathbf{f}$ gives rise to a cohomology class in $H^{n}(M, \mathbb{C} / \mathbb{Z})$. Denote by $\{\ln (\mathbf{f}, \gamma)\}$ its value on a cycle $\gamma$.

4.3. Multiplicative version of the logarithmic functional. One can define a multiplicative version of the logarithmic functional that takes a map $\mathbf{f}$ and a cycle $\gamma$ to the number $\exp (2 \pi i \ln (\mathbf{f}, \gamma))$. By the construction, the functional $\exp (2 \pi i \ln (\mathbf{f}, \gamma))$ takes values in $\mathbb{C}^{*}$ and has the following properties:

1 ) it depends skew-symmetrically on the components of $\mathbf{f}$, for example

$$
\exp \left(2 \pi i \ln \left(f_{1}, f_{2}, \ldots, f_{n+1}, \gamma\right)\right)=\left(\exp \left(2 \pi i \ln \left(f_{2}, f_{1}, \ldots, f_{n+1}, \gamma\right)\right)\right)^{(-1)}
$$

2 ) it depends multiplicatively on the components of $\mathbf{f}$, for example

$$
\begin{gathered}
\exp \left(2 \pi i \ln \left(\varphi_{1} \psi_{1}, f_{2}, \ldots, f_{n+1}, \gamma\right)\right)= \\
=\exp \left(2 \pi i \ln \left(\varphi_{1}, f_{2}, \ldots, f_{n+1}, \gamma\right) \exp 2 \pi i \ln \left(\psi_{1}, f_{2}, \ldots, f_{n+1}, \gamma\right)\right) .
\end{gathered}
$$


THEOREM. If the form $\mathbf{f}^{*} \omega$ is identically equal to zero on $M$, then the cochain, whose value on a cycle $\gamma$ equals to $\exp (2 \pi i\{\ln (\mathbf{f}, \gamma)\})$, is a cohomology class in $H^{n}\left(M, \mathbb{C}^{*}\right)$.

THEOREM. Let $X \subset\left(\mathbb{C}^{*}\right)^{n}$ be the real $n$-dimensional torus $\left|y_{1}\right|=\cdots=\left|y_{n}\right|=1$ and $\gamma$ the fundamental cycle of $X$. Consider $n+1$ monomials $c_{1} \mathbf{y}^{\mathbf{k}_{1}}, \ldots, c_{n+1} \mathbf{y}^{\mathbf{k}_{n+1}}$. Let $\mathbf{f}: X \rightarrow\left(\mathbb{C}^{*}\right)^{n+1}$ be the restriction of the $\operatorname{map}\left(c_{1} \mathbf{y}^{\mathbf{k}_{1}}, \ldots, c_{n+1} \mathbf{y}^{\mathbf{k}_{n+1}}\right):\left(\mathbb{C}^{*}\right)^{n} \rightarrow$ $\left(\mathbb{C}^{*}\right)^{n+1}$ to $X$. Then $\exp (2 \pi i\{\ln (\mathbf{f}, \gamma)\})$ is equal to the Parshin symbol $\left[c_{1} \mathbf{y}^{\mathbf{k}_{1}}, \ldots, c_{n+1} \mathbf{y}^{\mathbf{k}_{n+1}}\right]$.

THEOREM. Consider $n+1$ rational functions $\mathbf{f}=\left(f_{1}, \ldots, f_{n+1}\right)$ on an $n$ dimensional complete complex algebraic variety $X$. Let $F$ be a flag on $X$ and $\gamma_{F} \in H_{k}(U, \mathbb{Z})$ the flag-localized homology class. Then $\exp 2 \pi i\left\{\ln \left(\mathbf{f}, \gamma_{F}\right)\right\}$ is equal to the Parshin symbol $\left[f_{1}, \ldots, f_{n+1}\right]_{F}$.

The author would like to thank A.N. Parshin for his comments and T.V. Belokrinitskaya, V. Kirichenko and V.A. Timorin for their help in preparation of this paper.

\section{References}

1. Khovanskii A., Newton polytopes, curves on toric surfaces, and inversion of Weil's theorem, Russian Math. Surveys 52 (1997), no. 6, 1251-1279.

2. Beilinson A.A., Higher regulators and values of L-functions of curve, Functional analysis and Its Applications 14 (1980), no. 2, 116-118.

3. Deligne P., Le symbole modere., Publications Mathematiques de l'IHES, 73 (1991), 147-181.

4. Khovanskii A., Logarithmic functional and the Weil reciprocity law., Proceedings of the Waterloo Workshop on Computer Algebra 2006 (Kotsireas I, Zima E., eds.), World Scientific Press, 2007.

5. Khovanskii A., Newton polyhedrons, a new formula for mixed volume, product of roots of a system of equations, Proceed. of a Conf. in Honour of V.I. Arnold, vol. 24, Amer. Math. Soc., Fields Inst. Comm., USA, 1999, pp. 325-364.

6. Parshin A. N., Local class field theory, Trudy Mat. Inst. Steklov, vol. 165, 1984.

7. Parshin A. N., Galois cohomology and Brauer group of local fields, Trudy Mat. Inst. Steklov, vol. 183, 1990; English Transl. Proc. Steklov Inst. Math., vol 183, 191-201, 1991.

8. Fimmel T. and Parshin A. N., "Introduction to higher adelic theory", Preprint, Steklov Mathematical Institute, Moscow, 1999.

9. Khovanskii A., An Analog of Determinant Related to Parshin-Kato Theory and Integral Polytopes, Functional analysis and Its Applications 40 (2006), no. 2, 55-64.

10. Brylinski J.-L., McLaughlin D.A., Multidimensional reciprocity laws, J. reine angew. Math. 481 (1996), 125-147.

11. Khovanskii A., Multidimensional logarithmic functional and Parshin reciprocity laws., (in preparation). 